OPEN ACCESS

Edited by:

Gene W. Tyson,

University of Queensland, Australia

Reviewed by:

Hao Song,

Tianjin University, China

Christian Rinke,

University of Queensland, Australia

*Correspondence:

Edward F. DeLong

edelong@hawaii.edu

tThese authors have contributed equally to this work

Specialty section:

This article was submitted to Microbial Physiology and Metabolism,

a section of the journal

Frontiers in Microbiology

Received: 30 September 2015 Accepted: 25 January 2016

Published: 11 February 2016

Citation:

Mende DR, Aylward FO, Eppley JM,

Nielsen TN and DeLong EF (2016) Improved Environmental Genomes via Integration of Metagenomic and Single-Cell Assemblies.

Front. Microbiol. 7:143.

doi: 10.3389/fmicb.2016.00143

\section{Improved Environmental Genomes via Integration of Metagenomic and Single-Cell Assemblies}

\author{
Daniel R. Mende ${ }^{\dagger}$, Frank O. Aylward ${ }^{\dagger}$, John M. Eppley, Torben N. Nielsen and \\ Edward F. DeLong *
}

Daniel K. Inouye Center for Microbial Oceanography Research and Education, University of Hawai'i at Manoa, Honolulu, HI, USA

Assembling complete or near complete genomes from complex microbial communities remains a significant challenge in metagenomic studies. Recent developments in single cell amplified genomes (SAGs) have enabled the sequencing of individual draft genomes representative of uncultivated microbial populations. SAGs suffer from incomplete and uneven coverage due to artifacts that arise from multiple displacement amplification techniques. Conversely, metagenomic sequence data does not suffer from the same biases as SAGs, and significant improvements have been realized in the recovery of draft genomes from metagenomes. Nevertheless, the inherent genomic complexity of many microbial communities often obfuscates facile generation of population genome assemblies from metagenomic data. Here we describe a new method for metagenomicguided SAG assembly that leverages the advantages of both methods and significantly improves the completeness of initial SAGs assemblies. We demonstrate that SAG assemblies of two cosmopolitan marine lineages-Marine Group 1 Thaumarchaeota and SAR324 clade bacterioplankton-were substantially improved using this approach. Moreover, the improved assemblies strengthened biological inferences. For example, the improved SAR324 clade genome assembly revealed the presence of many genes in phenylalanine catabolism and flagellar assembly that were absent in the original SAG.

Keywords: metagenomics, single-cell genomics, SAGs, genome assembly, microbial oceanography

\section{INTRODUCTION}

An enormous amount of microbial biodiversity on Earth is comprised of lineages that cannot be cultivated using traditional techniques and have been largely inaccessible to laboratory analysis (Staley and Konopka, 1985; Pace, 1997; Hugenholtz et al., 1998). Despite their influence on global biogeochemical cycles and their ubiquity in diverse environments such as soils, the ocean, and metazoan hosts, much of the physiological and phylogenetic diversity of microbial "dark matter" remains unexplored (Rinke et al., 2013; Sharon and Banfield, 2013; Hedlund et al., 2014). Over the last few decades interest in uncultivated microbial diversity has steadily increased, and new methods have been developed for its discovery and characterization (Pace et al., 1986; Lasken, 2012; Stepanauskas, 2012; Blainey, 2013; Sharon and Banfield, 2013). Chief among these methodologies are metagenomics and single-cell genomics, which both enable for draft genomes of uncultivated

Abbreviations: MGI, Marine Group I; SAG, Single-cell Amplified Genome. 
phyla to be sequenced and their physiological potential to be analyzed without the need for extensive laboratory manipulation (Schmidt et al., 1991; Béjà et al., 2000; Stepanauskas, 2012; Sharon and Banfield, 2013).

Both metagenomics and single-cell genomics have independently led to major breakthroughs in our understanding of uncultivated microbial diversity, but both methods suffer from distinct limitations. Because deep coverage of highly related genotypes is generally necessary for genome assembly, metagenomics to date has been most successful in recovering genes and in some cases draft microbial genomes from abundant populations (Sharon and Banfield, 2013). Moreover, assembling genomes from many microbial communities and environments remains challenging due to high genomic richness and evenness that result in a lack of high genome coverage within any specific genotype (Hedlund et al., 2014). As an alternative, single-cell genomics can be leveraged to produce partial genome assemblies from microbial cells collected from complex environments, but assembling near-complete genomes is complicated by highly biased genome coverage resulting from multiple displacement amplification (MDA; Hedlund et al., 2014). The draft genomes that result from these two different approaches are also qualitatively distinct; genomes resulting from metagenomic analyses represent "composite genomes" that incorporate genetic information from genotypically heterogeneous populations, while single cell amplified genomes (SAGs) represent genomes of individual cells that may or may not contain the full genetic repertoire present in their larger sympatric populations.

The distinct challenges and common goals of metagenomics and single-cell genomics make these technologies synergistic (Lasken, 2012; Hedlund et al., 2014), and several studies have already leveraged both methods. One study analyzing ammonia oxidation in San Francisco Bay generated five singlecell genomes and one metagenome from an enrichment culture and combined all of these sequencing datasets to yield a draft genome of the low-salinity ammonia-oxidizing archaea 'Candidatus Nitrosoarchaeum limnia SFB1' (Blainey et al., 2011). Other studies have leveraged both methods for comparative genomics; two recent studies have compared SAGs and metagenome-derived composite genomes from the candidate phylum "Atribacteria" to analyze its fermentative metabolism (Dodsworth et al., 2013; Nobu et al., 2015a), while another analyzed several SAGs and composite metagenomic assemblies of the ubiquitous SAR86 clade of marine bacterioplankton (Dupont et al., 2011). In all of these cases comparison of the draft genomes generated with different techniques allowed for novel biological insights to be drawn. Other studies have also used single-cell genomes as scaffolds for comparison or recruitment of metagenomic data when appropriate reference genomes would otherwise be unavailable, allowing for more robust analysis of metagenomic data (Eloe et al., 2011; Hess et al., 2011; Swan et al., 2013; Roux et al., 2014; Nobu et al., 2015b).

Given the overlapping goals of metagenomics and single-cell genomics, we anticipate that studies using both methodologies will become more common in the future. To facilitate the integration of these methods we developed a workflow for the combination of single-cell genomic and metagenomic data that can be used to assemble improved draft genomes from environmental samples. We present a systematic and generalized methodology that leverages this integrated approach to improve SAG genome assemblies and discuss the potential of this technique for future investigations.

\section{MATERIALS AND METHODS}

\section{Data Acquisition}

Metagenomic sequencing data was generated from a sample taken on November 29th at a depth of $500 \mathrm{~m}$ from Station ALOHA on cruise \#237 of the Hawaii Ocean Time-series (HOT). Metadata for this cruise is available on the website for the Hawaii Ocean Time-series Data Organization and Graphical System (HOT-DOGS) at http://hahana.soest.hawaii.edu/hot/hot-dogs/.

The two liters of water collected were pre-filtered with a $1.6 \mu \mathrm{m} 42.5 \mathrm{~mm}$ Whatman GFA filter (Cat. No. 1820-042, Whatman) and filtrate was collected on $0.22 \mu \mathrm{m}$ sterivex GV filter for DNA (Cat No: SVGV01015, Millipore). Cells were lysed with sucrose lysis buffer [40 mM EDTA, $50 \mathrm{mM}$ Tris ( $\mathrm{pH} 8.3$ ), $0.75 \mathrm{M}$ Sucrose] containing $2 \mathrm{mg} / \mathrm{ml}$ of lysozyme incubated at $37^{\circ} \mathrm{C}$ for $30 \mathrm{~min}$. Final concentrations of $1 \%$ SDS and $0.75 \mathrm{mg} / \mathrm{ml}$ Proteinase $\mathrm{K}$ was added and solution was incubated for $2 \mathrm{~h}$ at $55^{\circ} \mathrm{C}$. DNA purification was performed using the FujiFilm Quick Gene instrument with the QuickGene DNA Tissue Kit (Cat. No DT-L Life Science). Libraries were created using the Illumina TruSeq LT Nano kit set A (PN: FC-121-4001). Sequencing data was generated using an Illumina MiSeq system, producing 43,359,550 individual 300 bp reads.

In this study we analyzed three SAGs generated from a clade SAR324 bacterioplankton (SAR324 cluster bacterium SCGC AAA240-J09), a clade SAR11 bacterioplankton (alpha proteobacterium SCGC AAA240-E13), and a MGI Thaumarchaeota (Thaumarchaeota archaeon SCGC AAA007O23). The SAR324 and SAR11 SAGs were both recovered from samples taken at Station ALOHA at a depth of $770 \mathrm{~m}$ (Swan et al., 2011; Thrash et al., 2014), while the MGI Thaumarchaeota was recovered from a sample taken in the South Atlantic (Swan et al., 2014). We obtained the raw Illumina sequencing data for these SAGs from the DOE-JGI Genome Portal website (http:// genome.jgi.doe.gov/), while the published assemblies for these SAGs were obtained from NCBI GenBank (Benson et al., 2013).

\section{Metagenome and SAG Assembly}

For both SAG and metagenomic raw data we quality filtered all raw reads using MIRA (version: 4.9.5_2) with the qc and pec options and standard parameters to retain a "high confidence region" (HCR) of every read. This step also includes the removal of contamination by phiX (Chevreux, 2004). Quality filtered SAG sequencing data was assembled using the SPAdes genome assembly program (version v.3.5.0) with default parameters (Figure 1, Step 1). SPAdes was chosen as an assembler as it is specifically designed for handling SAG sequencing data (Bankevich et al., 2012). For quality-trimmed metagenomic data we used MIRA (version: 4.9.5_2) to assemble metagenomic data using the standard workflow for accurate de 


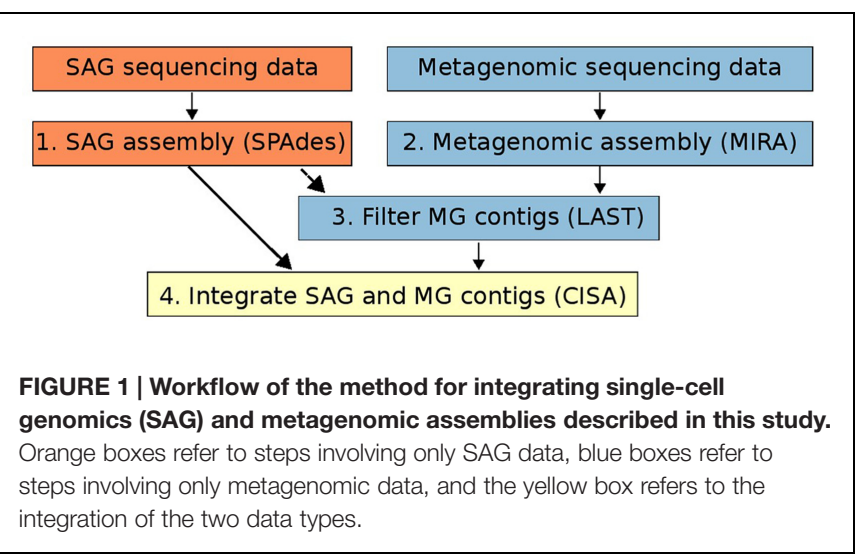

novo genome assembly (Chevreux, 2004; Figure 1, Step 2). For both SAG and metagenomic assemblies only contigs longer than $1 \mathrm{kbp}$ were retained for downstream processing. Although we suggest SPAdes and Mira for SAG and metagenome assembly, respectively, in principle the assembly integration workflow presented here can be used with assemblies generated from any program (also see Figure 1).

\section{Integration of SAG and Metagenomic Assemblies}

Metagenomic contigs were aligned to the newly assembled SAG contigs using LAST (Kielbasa et al., 2011) and were extracted if they matched at $>95 \%$ nucleotide identity over more than 200 bp (Figure 1, Step 3). These contigs are putatively from the same organism or population as the SAG contigs (Konstantinidis and Tiedje, 2005). Next, we used CISA (Lin and Liao, 2013) to combine the extracted metagenomic and the SAG contigs into an integrated assembly (Figure 1, Step 4). For each newly generated contig, CISA reports which assembly provided the backbone. In a final filtering step, only contigs whose backbone was based on the SAG assembly were kept for the final improved SAG (iSAG) assembly. We used checkM (Parks et al., 2015) to assess both the completeness and contamination of the original and improved SAGs. Moreover, we also used the ProDeGe tool (Tennessen et al., 2015) to assess the degree of contamination both in the original SAGs analyzed and the finalized iSAGs. The results are summarized in Table $\mathbf{1}$.

\section{Read Mapping}

To assess the extent to which sequences highly similar to the SAGs of interest were present in the metagenomic data we mapped metagenomic reads against the original SAG assemblies using LAST (Kielbasa et al., 2011) with default parameters. We visualized the results with fragment recruitment plots (Rusch et al., 2007) generated from the mapping data using the ggplot 2 package from the $\mathrm{R}$ statistical programming environment ( $\mathrm{R}$ Development Core Team, 2011; Figure 2). From these plots, the suitability of a metagenome for this workflow can be confirmed (as in the case of the SAR324 and MGI Thaumarchaeota SAGs) or refuted (as in the case of the SAR11 SAG; Figure 2C). Further, we assessed the similarity between the iSAGs and both the metagenomic sequencing reads as well as the SAG sequencing reads. For this purpose we aligned all sequencing reads using LAST and generated fragment recruitment plots (for the metagenome mapping) and density plots (for the raw SAG read mapping) to visualize these alignments (Figure 3).

\section{Functional Annotations}

Annotations for the SAR324 SAG and iSAG were performed using the KEGG Automatic Annotation Server (KAAS; Moriya et al., 2007; Kanehisa et al., 2008). All complete and

TABLE 1 | Comparison of assembly statistics of the original single-cell genomics (SAGs) and iSAGs presented here for SAR324 and MGI Thaumarchaeota.

\begin{tabular}{|c|c|c|c|c|}
\hline & \multicolumn{2}{|c|}{ SAR324 } & \multicolumn{2}{|c|}{ MGI Thaumarchaeota } \\
\hline & Original SAG & iSAG & Original SAG & iSAG \\
\hline Completeness & 43.67 & 65.78 & 96.88 & 96.88 \\
\hline Genome size (bp) & $2,264,488$ & $2,379,063$ & $1,104,470$ & $1,093,884$ \\
\hline \#Contigs & 672 & 13 & 32 & 4 \\
\hline N50 (contigs) & 22,317 & 191,983 & 79,020 & 313,273 \\
\hline Longest contig (bp) & 94,006 & 354,247 & 217,386 & 319,413 \\
\hline GC & 41.49 & 42.59 & 35.66 & 35.61 \\
\hline GC std (contigs > 1 kbp) & 3.98 & 0.76 & 2.1 & 0.75 \\
\hline Coding density & 87.03 & 89.67 & 92.87 & 93.11 \\
\hline \#Predicted genes & 2,533 & 2,137 & 1,356 & 1,298 \\
\hline \#Complete genes & 1,827 & 2,120 & 1,307 & 1,290 \\
\hline \#Missing marker genes & 84 & 50 & 4 & 4 \\
\hline \#Marker genes in single copy & 104 & 141 & 138 & 142 \\
\hline \#Marker genes found multiple times & 3 & 0 & 4 & 0 \\
\hline Contamination (checkM) (\%) & 0.64 & 0 & 2.07 & 0 \\
\hline Contamination (ProDeGe): (\% Contigs) & 89.88 & 0 & 40.63 & 0 \\
\hline
\end{tabular}

Std, standard deviation. 


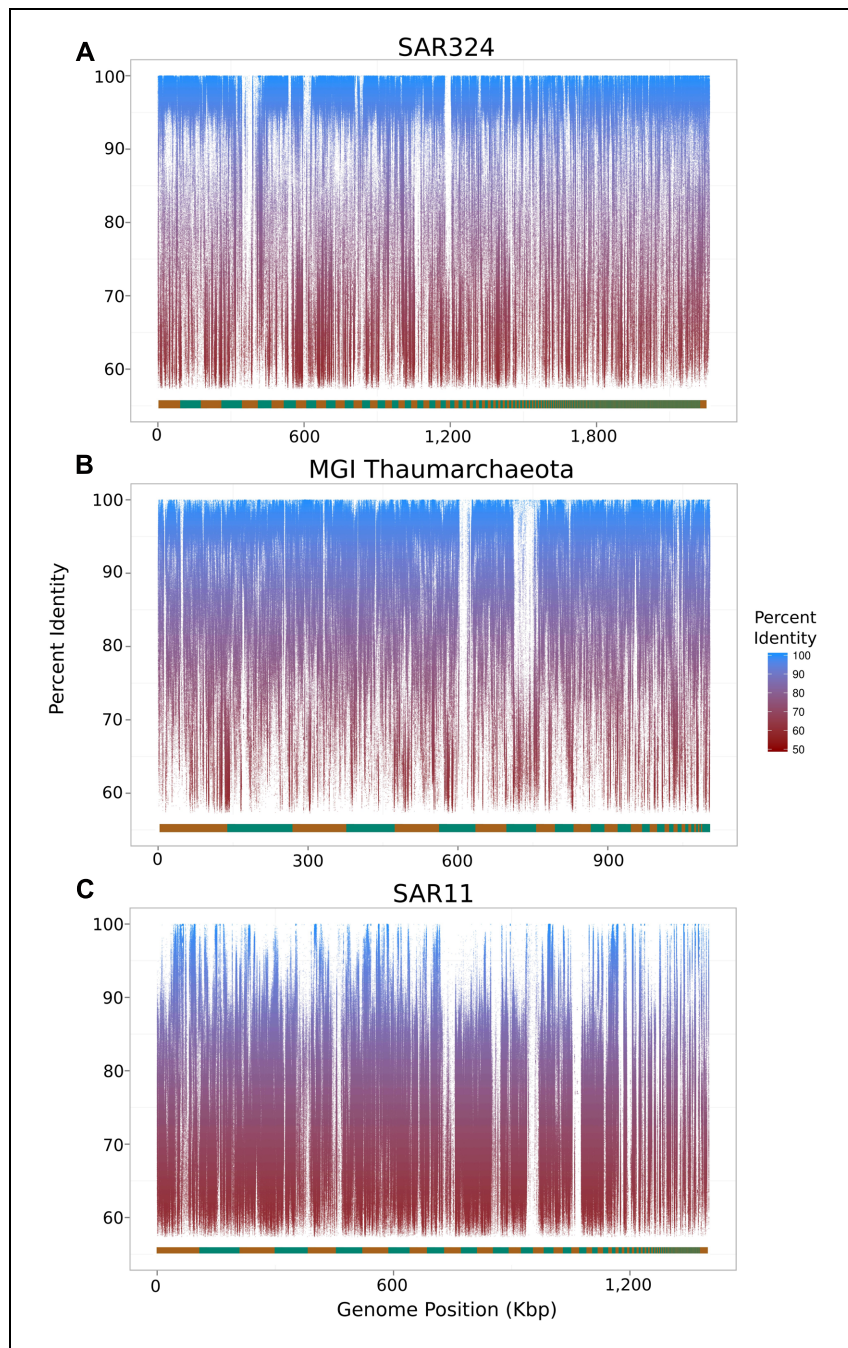

FIGURE 2 | Fragment recruitment plots of metagenomic reads mapped onto the original SAR324 clade bacterioplankton (A), MGI Thaumarchaeota (B), and SAR11 clade bacterioplankton (C) SAGs analyzed in this study. The alternating orange and green bar at the bottom of each plot shows the contig boundaries, which are ordered from longest to shortest.

partial proteins predicted from these genomes were used for annotations.

\section{RESULTS AND DISCUSSION}

In this study we designed a workflow to combine SAGs with metagenomic data from the same environment to produce integrated, higher-quality genomes we refer to here as "improved SAGs", or iSAGs (Figure 1, also see Materials and Methods). We analyzed a deeply sequenced metagenome from mesopelagic waters of Station ALOHA in the North Pacific Subtropical Gyre (presented here) together with three previously published SAGs sampled from mesopelagic waters in the Pacific and Atlantic oceans that represent the marine SAR11 clade of Alphaproteobacteria (Thrash et al., 2014), the
SAR324 clade of Deltaproteobacteria (Swan et al., 2011), and MGI Thaumarchaeota (Swan et al., 2014). The success of our method varied across the three SAGs analyzed, and overall our results provide useful examples of the advantages and limitations of using metagenomic data for the improvement of assemblies generated from single-cell sequencing.

Our workflow was successful in producing iSAGs for both the SAR324 and MGI Thaumarchaeota genomes. Compared to the original SAGs, the iSAGs for these two groups were consolidated into fewer contigs, displayed increased N50 contig lengths, and contained fewer duplicate marker genes that are indicative of contamination or mis-assembly (Table 1). The SAR324 iSAG showed particular improvement, with an estimated $22.1 \%$ increase in completeness as estimated from conserved marker genes (65.8\% from $43.7 \%$ ) and an additional $115 \mathrm{~Kb}$ of sequence present when compared to the original SAG. The MGI Thaumarchaeota SAG was already nearly complete prior to our analysis (96\%), but our workflow succeeded in consolidating the original 32 contigs into just 4, increasing the N50 contig size from 79 to $313 \mathrm{~Kb}$, and reducing the estimated contamination of the contigs from $2 \%$ to $0 \%$. Moreover, in both the SAR324 and MGI Thaumarchaeota the total number of predicted genes was reduced in the iSAG compared to the original SAG, although the total number of complete genes increased in the SAR324 iSAG (SAG: 1,827, iSAG: 2,120) and stayed relatively constant in the MGI Thaumarchaeota (SAG: 1,307, iSAG: 1,290). This reduction in fragmented genes is likely due to the consolidation of the genomes into fewer contigs, the removal of contigs $<1 \mathrm{~Kb}$, and the removal of redundancy and contamination, providing for more robust gene prediction and annotation.

For the SAR11 SAG analyzed, we were unable to produce an improved assembly since genotypes bearing high similarity to the original SAR11 SAG were absent, or not present in high abundance, in our metagenomic sample. Fragment recruitment plots visualizing alignments of metagenomic reads onto the three SAGs revealed that the vast majority of the SAR324 and MGI Thaumarchaeota SAGs contained highidentity matches to sequences in the metagenomic data, while this was true for only very few regions of the SAR11 SAG (Figure 2). This lack of representation of the SAR11 SAG in the metagenome underscores the importance of selecting SAGs that belong to populations well-represented in the cognate metagenomic data for the approach described here to be successful. Optimally, of course, the SAGs and the metagenome should be derived from the same sample population. It is possible that the SAR11 SAG analyzed here may belong to a low-abundance population that was not sampled to sufficient depth in the metagenomic data for improved assemblies to be possible. Moreover, it should be noted that in this study the metagenomes and the SAR11 and SAR324 SAGs were sampled from mesopelagic waters of Station ALOHA $(770 \mathrm{~m})$ at different time points, while the MGI Thaumarchaeota SAG was sampled from mesopelagic waters of the South Atlantic $(800 \mathrm{~m})$. Interestingly, although we were unable to improve the SAR11 SAG with our metagenomic data (both sampled at Station ALOHA in the Pacific; the former at $770 \mathrm{~m}$ and the latter at $500 \mathrm{~m}$ ), we did succeed in improving 


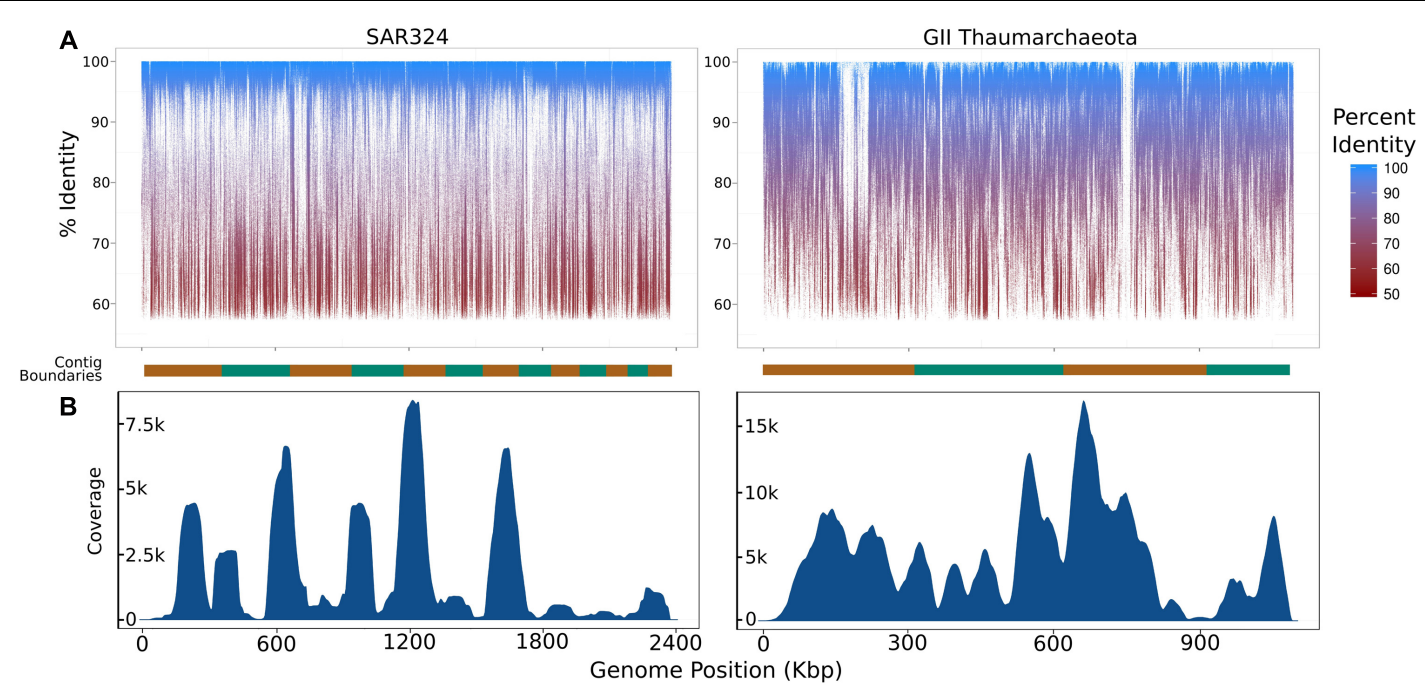

FIGURE 3 | Summary of coverage of the iSAGs assembled here in the metagenomic and single-cell datasets used. (A) Fragment recruitment plots of metagenomic reads mapped onto the iSAGs constructed in this study. The alternating orange and green bar at the bottom of each plot shows the contig boundaries, which are ordered from longest to shortest. (B) Density plots showing the relative coverage of the raw SAG reads mapped onto the iSAGs constructed here.

the MGI Thaumarchaeota genome from the Atlantic with the same metagenomic data. We speculate that this is due to a combination of a low degree of genetic diversity in Thaumarchaeota populations, as suggested by previous studies (Hallam et al., 2006; Stieglmeier et al., 2014), in conjunction with a high abundance of this group in our metagenomic data. Despite the success of improving the MGI Thaumarchaeota SAG, however, we anticipate that the method described here would be in general most effective if used with metagenomic and SAG data sequenced from the same environment at the same time.

To further analyze the iSAGs we mapped reads from both the metagenomic data and the original SAG data back onto the new assemblies to visualize their representation in these two datasets. Similar to the mapping of metagenomic reads onto the original SAGs, mapping of this data onto the iSAGs revealed high identity matches across the majority of both genomes, with relatively few gaps (Figure 3A). The gaps present in the metagenome fragment recruitment plots may correspond to genomic islands that are either absent or present in low abundance in the populations sampled in the metagenomic data. This is likely to be the case for the two large gaps observed in the MGI Thaumarchaeota, especially when considering that the original SAG and metagenomic data were obtained from different water masses and likely represent distinct, albeit highly similar, population genotypes. We note that the mapping of metagenomic data back onto improved SAGs could be potentially applied to analyze population heterogeneity in a single sample. Because SAGs represent the genome of an individual cell, they may include low-abundance variants not well-represented in metagenomic data. The inclusion of both data types thus provides a more complete picture of genomic diversity in nature.
In contrast to the metagenome fragment recruitment plots, density plots showing the results of mapping SAG reads onto the iSAGs highlight the large variation in coverage typical of SAG sequencing projects (Figure 3B). The use of MDA in the preparation of SAGs amplifies regions inconsistently across a genome, which produces highly variable coverage in the resulting sequencing data that is a major impediment to subsequent assembly (Lasken, 2012; Hedlund et al., 2014). Our ability to improve the original SAR324 and MGI Thaumarchaeota SAG assemblies demonstrates that the inclusion of metagenomic data, which contains much less variability in coverage, allows for the joining and consolidation of many smaller contigs produced from SAG assembly alone. Use of both datasets together thus mitigates the effects of the low coverage regions biased against by MDA while making full use of the larger contigs afforded by the higher coverage regions.

To investigate if improvement of the SAG data as described here can improve biological inferences, we compared the coding potential of the original SAR324 SAG to that of the iSAG. We focused our analyses on the SAR324 genome because it showed the largest improvement in our completeness analysis (Table 1). Comparison of both SAR324 SAG and iSAG to the Kyoto Encyclopedia of Genes and Genomes (Kanehisa et al., 2008) revealed that many metabolic pathways were more complete in the iSAG (Table 2). Two of these pathways, those for Flagellar Assembly and Phenylalanine Degradation, showed particular improvement, and are displayed in Figure 4. For Flagellar Assembly the original SAR324 SAG encoded only 13 of the 32 genes $(40.6 \%)$ in this pathway while the iSAG encoded $23(71.9 \%)$. In the case of Phenylalanine Degradation, the original SAG encoded only genes for 3 of the 11 steps $(27.3 \%)$ in this pathway, while the iSAG encoded the genes necessary for 10 of the 11 (90.9\%; including two genes that 
TABLE 2 | Comparison of KEGG Orthology protein annotations of the original SAR324 SAG and the iSAG presented in this paper.

\begin{tabular}{|c|c|c|c|}
\hline Pathway & SAR324 SAG & SAR324 iSAG & New genes identified \\
\hline Biosynthesis of amino acids & 76 & 92 & 16 \\
\hline Phenylalanine metabolism & 7 & 21 & 14 \\
\hline Flagellar assembly & 13 & 23 & 10 \\
\hline Carbon metabolism & 59 & 68 & 9 \\
\hline Two-component system & 23 & 32 & 9 \\
\hline Oxidative phosphorylation & 21 & 29 & 8 \\
\hline Purine metabolism & 40 & 48 & 8 \\
\hline Cysteine and methionine metabolism & 15 & 23 & 8 \\
\hline Ribosome & 31 & 39 & 8 \\
\hline Glyoxylate and dicarboxylate metabolism & 14 & 21 & 7 \\
\hline Carbon fixation pathways in prokaryotes & 16 & 23 & 7 \\
\hline Aminoacyl-tRNA biosynthesis & 16 & 23 & 7 \\
\hline 2-Oxocarboxylic acid metabolism & 12 & 18 & 6 \\
\hline Pyrimidine metabolism & 26 & 32 & 6 \\
\hline Phenylalanine, tyrosine and tryptophan biosynthesis & 15 & 21 & 6 \\
\hline Folate biosynthesis & 4 & 10 & 6 \\
\hline Glycerophospholipid metabolism & 6 & 11 & 5 \\
\hline Glycine, serine and threonine metabolism & 24 & 29 & 5 \\
\hline Ubiquinone and terpenoid-quinone biosynthesis & 4 & 9 & 5 \\
\hline Propanoate metabolism & 9 & 13 & 4 \\
\hline Protein-coding genes with $\mathrm{KO}$ annotations & 964 & 1,173 & 209 \\
\hline
\end{tabular}

encode the proteins PaaJ and PaaZ that each catalyze two reactions of the pathway). Other pathways were also more complete in the SAR324 iSAG, including those for amino acid biosynthesis, carbon metabolism, oxidative phosphorylation, and other vitamin and nucleotide biosynthetic processes (Table 2). Additionally, the total number of genes that could be annotated in KEGG was increased in the iSAG as compared to the SAG (SAG: 965, iSAG:1,173), consistent with our results of fewer fragmented genes in the iSAG and previous findings that consolidation of a genome into fewer contigs leads to improved gene prediction and annotation (Klassen and Currie, 2012).

In this study we present a novel method for the generation of improved assemblies of environmental genomes using a combination of SAGs and metagenomic data. Results from the three SAGs analyzed in this work provide different examples of how this method performs. In the example of the SAR324 SAG we observed the largest improvement, with a significant increase in completeness, reduction in the total number of contigs, and enhanced recovery of genes and metabolic pathways. In the case of the MGI Thaumarchaeota, although the initial SAG was already near-complete, we were still able to consolidate the total number of contigs to just 4 and remove several genomic regions representing potential contamination. This was somewhat surprising given the MGI Thaumarchaeota SAG originated from the South Atlantic while the metagenomic sample originated from the North Pacific. This suggests that for population genotypes with high conservation and broad distributions the judicious application of this approach may even be used across disparate samples. Finally, the SAR11 SAG used in this study could not be improved due to a lack of abundant and highly similar genotypes in the metagenomic datasets we used, demonstrating that this method necessarily has stringent requirements for high population genotype similarity ( $>95 \%$ ANI) between the SAGs and metagenomes utilized. Ideally, both SAGs and metagenomes should derive from the same or similar sample, albeit for conserved population genotypes this may not be an absolute requirement. The addition of longer reads derived from newer sequencing technologies (Koren et al., 2012; McCoy et al., 2014; Goodwin et al., 2015) will likely provide additional improvement to genome assemblies in the future. Due to the widespread interest in uncultivated microbial groups in the biosphere as well as the valuable information that can be gleaned from improved genome assemblies, we anticipate that workflows such as the one described here will be a useful addition to the -omics methods currently available to researchers.

\section{Data Availability}

Raw reads generated from metagenome sequencing are available in the NCBI Short Read Archive under accession number SRP066631. The iSAGs generated as part of this study are available in the Supplementary Information.

\section{AUTHOR CONTRIBUTIONS}

DM and FA designed the research, performed the analysis and wrote the manuscript. JE and TN performed the sequence assemblies and revised the manuscript critically. ED designed the research and wrote the manuscript. 


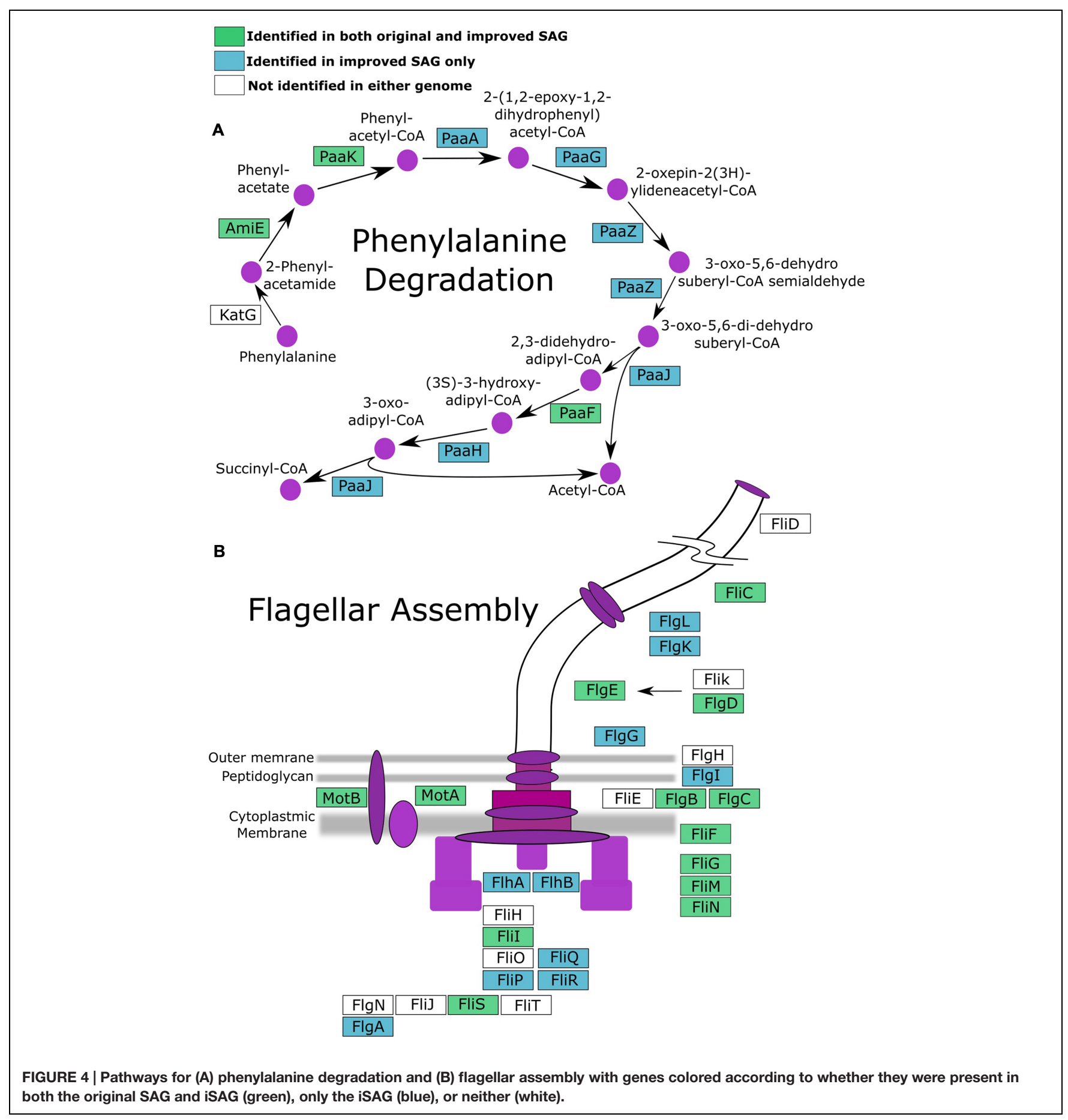

\section{FUNDING}

This paper is a contribution from the Center for Microbial Oceanography Research and Education (C-MORE) and the Simons Collaboration on Ocean Processes and Ecology (SCOPE). This work was supported by grants from the Gordon and Betty Moore Foundation (to ED, 3777), National Science Foundation Grant EF0424599 (to ED), and the Simons Foundation grant Simons Collaboration on Ocean Processes and Ecology (SCOPE) (to ED, 329108). DM was supported by SCOPE, EMBO (ALTF 721-2015), and the European Commission (LTFCOFUND2013, GA-2013-609409).

\section{ACKNOWLEDGMENTS}

We thank all members of the Hawaii Ocean Time-series team for sample collection, and the crew of the $\mathrm{R} / \mathrm{V}$ 
Ka'imikai-o-Kanaloa for shipboard operations during HOT cruise 237. Further, we thank T. Palden and A. Romano for processing the metagenomic sample and helpful comments. We thank two reviewers for their helpful comments.

\section{REFERENCES}

Bankevich, A., Nurk, S., Antipov, D., Gurevich, A. A., Dvorkin, M., Kulikov, A. S., et al. (2012). SPAdes: a new genome assembly algorithm and its applications to single-cell sequencing. J. Comput. Biol. 19, 455-477. doi: $10.1089 / \mathrm{cmb} .2012 .0021$

Béjà, O., Aravind, L., Koonin, E. V., Suzuki, M. T., Hadd, A., Nguyen, L. P., et al. (2000). Bacterial rhodopsin: evidence for a new type of phototrophy in the sea. Science 289, 1902-1906. doi: 10.1126/science.289.5486.1902

Benson, D. A., Cavanaugh, M., Clark, K., Karsch-Mizrachi, I., Lipman, D. J., Ostell, J., et al. (2013). GenBank. Nucleic Acids Res. 41, D36-D42. doi: $10.1093 / \mathrm{nar} / \mathrm{gks} 1195$

Blainey, P. C. (2013). The future is now: single-cell genomics of bacteria and archaea. FEMS Microbiol. Rev. 37, 407-427. doi: 10.1111/1574-6976.12015

Blainey, P. C., Mosier, A. C., Potanina, A., Francis, C. A., and Quake, S. R. (2011). Genome of a low-salinity ammonia-oxidizing archaeon determined by single-cell and metagenomic analysis. PLOS ONE 6:e16626. doi: 10.1371/journal.pone.0016626

Chevreux, B. (2004). Using the miraEST assembler for reliable and automated mRNA transcript assembly and snp detection in sequenced ESTs. Genome Res. 14, 1147-1159. doi: 10.1101/gr.1917404

Dodsworth, J. A., Blainey, P. C., Murugapiran, S. K., Swingley, W. D., Ross, C. A., Tringe, S. G., et al. (2013). Single-cell and metagenomic analyses indicate a fermentative and saccharolytic lifestyle for members of the OP9 lineage. Nat. Commun. 4:1854. doi: 10.1038/ncomms2884

Dupont, C. L., Rusch, D. B., Shibu, Y., Mary-Jane, L., Alexander Richter, R., Ruben, V., et al. (2011). Genomic insights to SAR86, an abundant and uncultivated marine bacterial lineage. ISME J. 6, 1186-1199. doi: 10.1038/ismej.2011.189

Eloe, E. A., Fadrosh, D. W., Novotny, M., Zeigler Allen, L., Kim, M., Lombardo, M.-J., et al. (2011). Going deeper: metagenome of a hadopelagic microbial community. PLOS ONE 6:e20388. doi: 10.1371/journal.pone. 0020388

Goodwin, S., Sara, G., James, G., Scott, E.-S., Panchajanya, D., Michael, S., et al. (2015). Oxford nanopore sequencing, hybrid error correction, and de novo assembly of a eukaryotic genome. Genome Res. 25, 1750-1756. doi: $10.1101 /$ gr.191395.115

Hallam, S. J., Konstantinidis, K. T., Putnam, N., Schleper, C., Watanabe, Y.-I., Sugahara, J., et al. (2006). Genomic analysis of the uncultivated marine crenarchaeote Cenarchaeum symbiosum. Proc. Natl. Acad. Sci. U.S.A. 103, 18296-18301. doi: 10.1073/pnas.0608549103

Hedlund, B. P., Dodsworth, J. A., Murugapiran, S. K., Rinke, C., and Woyke, T. (2014). Impact of single-cell genomics and metagenomics on the emerging view of extremophile "microbial dark matter." Extremophiles 18, 865-875. doi: 10.1007/s00792-014-0664-7

Hess, M., Sczyrba, A., Egan, R., Kim, T.-W., Chokhawala, H., Schroth, G., et al. (2011). Metagenomic discovery of biomass-degrading genes and genomes from cow rumen. Science 331, 463-467. doi: 10.1126/science.1200387

Hugenholtz, P., Goebel, B. M., and Pace, N. R. (1998). Impact of cultureindependent studies on the emerging phylogenetic view of bacterial diversity. J. Bacteriol. 180, 4765-4774.

Kanehisa, M., Araki, M., Goto, S., Hattori, M., Hirakawa, M., Itoh, M., et al. (2008). KEGG for linking genomes to life and the environment. Nucleic Acids Res. 36, D480-D484. doi: 10.1093/nar/gkm882

Kielbasa, S. M., Wan, R., Sato, K., Horton, P., and Frith, M. C. (2011). Adaptive seeds tame genomic sequence comparison. Genome Res. 21, 487-493. doi: $10.1101 /$ gr.113985.110

Klassen, J. L., and Currie, C. R. (2012). Gene fragmentation in bacterial draft genomes: extent, consequences and mitigation. BMC Genomics 13:14. doi: 10.1186/1471-2164-13-14

\section{SUPPLEMENTARY MATERIAL}

The Supplementary Material for this article can be found online at: http://journal.frontiersin.org/article/10.3389/fmicb. 2016.00143

Konstantinidis, K. T., and Tiedje, J. M. (2005). Genomic insights that advance the species definition for prokaryotes. Proc. Natl. Acad. Sci. U.S.A. 102, 2567-2572. doi: 10.1073/pnas.0409727102

Koren, S., Schatz, M. C., Walenz, B. P., Martin, J., Howard, J. T., Ganapathy, G., et al. (2012). Hybrid error correction and de novo assembly of single-molecule sequencing reads. Nat. Biotechnol. 30, 693-700. doi: 10.1038/nbt.2280

Lasken, R. S. (2012). Genomic sequencing of uncultured microorganisms from single cells. Nat. Rev. Microbiol. 10, 631-640. doi: 10.1038/nrmicro 2857

Lin, S.-H., and Liao, Y.-C. (2013). CISA: contig integrator for sequence assembly of bacterial genomes. PLoS ONE 8:e60843. doi: 10.1371/journal.pone.00 60843

McCoy, R. C., Taylor, R. W., Blauwkamp, T. A., Kelley, J. L., Kertesz, M., Pushkarev, D., et al. (2014). Illumina TruSeq synthetic long-reads empower de novo assembly and resolve complex, highly-repetitive transposable elements. PLoS ONE 9:e106689. doi: 10.1371/journal.pone.0106689

Moriya, Y., Itoh, M., Okuda, S., Yoshizawa, A. C., and Kanehisa, M. (2007). KAAS: an automatic genome annotation and pathway reconstruction server. Nucleic Acids Res. 35, W182-W185. doi: 10.1093/nar/gkm321

Nobu, M. K., Dodsworth, J. A., Murugapiran, S. K., Rinke, C., Gies, E. A., Webster, G., et al. (2015a). Phylogeny and physiology of candidate phylum "Atribacteria" (OP9/JS1) inferred from cultivation-independent genomics. ISME J. 10, 273-286. doi: 10.1038/ismej.2015.97

Nobu, M. K., Takashi, N., Christian, R., Yoichi, K., Tringe, S. G., Tanja, W., et al. (2015b). Microbial dark matter ecogenomics reveals complex synergistic networks in a methanogenic bioreactor. ISME J. 9, 1710-1722. doi: 10.1038/ismej.2014.256

Pace, N. R. (1997). A molecular view of microbial diversity and the biosphere. Science 276, 734-740. doi: 10.1126/science.276.5313.734

Pace, N. R., Stahl, D. A., Lane, D. J., and Olsen, G. J. (1986). “The analysis of natural microbial populations by ribosomal RNA sequences," in Advances in Microbial Ecology, ed. K. C. Marshall (New York: Springer US), 1-55.

Parks, D. H., Michael, I., Skennerton, C. T., Philip, H., and Tyson, G. W. (2015). CheckM: assessing the quality of microbial genomes recovered from isolates, single cells, and metagenomes. Genome Res. 25, 1043-1055. doi: 10.1101/gr.186072.114

R Development Core Team (2011). R: A Language and Environment for Statistical Computing. Vienna: R Development Core Team.

Rinke, C., Schwientek, P., Sczyrba, A., Ivanova, N. N., Anderson, I. J., Cheng, J.-F., et al. (2013). Insights into the phylogeny and coding potential of microbial dark matter. Nature 499, 431-437. doi: 10.1038/nature12352

Roux, S., Simon, R., Hawley, A. K., Beltran, M. T., Melanie, S., Patrick, S., et al. (2014). Ecology and evolution of viruses infecting uncultivated SUP05 bacteria as revealed by single-cell- and meta-genomics. eLife 3:e03125. doi: 10.7554/elife. 03125

Rusch, D. B., Halpern, A. L., Sutton, G., Heidelberg, K. B., Williamson, S., Yooseph, S., et al. (2007). The Sorcerer II global ocean sampling expedition: northwest atlantic through eastern tropical Pacific. PLoS Biol. 5:e77. doi: 10.1371/journal.pbio.0050077

Schmidt, T. M., DeLong, E. F., and Pace, N. R. (1991). Analysis of a marine picoplankton community by $16 \mathrm{~S}$ rRNA gene cloning and sequencing. J. Bacteriol. 173, 4371-4378.

Sharon, I., and Banfield, J. F. (2013). Microbiology. Genomes from metagenomics. Science 342, 1057-1058. doi: 10.1126/science.1247023

Staley, J. T., and Konopka, A. (1985). Measurement of in situ activities of nonphotosynthetic microorganisms in aquatic and terrestrial habitats. Annu. Rev. Microbiol. 39, 321-346. doi: 10.1146/annurev.mi.39.100185. 001541

Stepanauskas, R. (2012). Single cell genomics: an individual look at microbes. Curr. Opin. Microbiol. 15, 613-620. doi: 10.1016/j.mib.2012.09.001 
Stieglmeier, M., Michaela, S., Alves, R. J. E., and Christa, S. (2014). “The phylum Thaumarchaeota," in The Prokaryotes, Other Major Lineages of Bacteria and the Archaea, eds E. Rosenberg, E. F. DeLong, S. Lory, E. Stackebrandt, and F. Thompson (Berlin: Springer-Verlag Berlin Heidelberg), 347-362.

Swan, B. K., Chaffin, M. D., Manuel, M.-G., Morrison, H. G., Field, E. K., Poulton, N. J., et al. (2014). Genomic and metabolic diversity of marine group I thaumarchaeota in the mesopelagic of two subtropical gyres. PLOS ONE 9:e95380. doi: 10.1371/journal.pone.0095380

Swan, B. K., Martinez-Garcia, M., Preston, C. M., Sczyrba, A., Woyke, T., Lamy, D., et al. (2011). Potential for chemolithoautotrophy among ubiquitous bacteria lineages in the dark ocean. Science 333, 1296-1300. doi: 10.1126/science. 1203690

Swan, B. K., Tupper, B., Sczyrba, A., Lauro, F. M., Martinez-Garcia, M., González, J. M., et al. (2013). Prevalent genome streamlining and latitudinal divergence of planktonic bacteria in the surface ocean. Proc. Natl. Acad. Sci. U.S.A. 110, 11463-11468. doi: 10.1073/pnas.1304246110

Tennessen, K., Andersen, E., Clingenpeel, S., Rinke, C., Lundberg, D. S., Han, J., et al. (2015). ProDeGe: a computational protocol for fully automated decontamination of genomes. ISME J. 10, 269-272. doi: 10.1038/ismej. 2015.100

Thrash, J. C., Temperton, B., Swan, B. K., Landry, Z. C., Woyke, T., DeLong, E. F., et al. (2014). Single-cell enabled comparative genomics of a deep ocean SAR11 bathytype. ISME J. 8, 1440-1451. doi: 10.1038/ismej. 2013.243

Conflict of Interest Statement: The authors declare that the research was conducted in the absence of any commercial or financial relationships that could be construed as a potential conflict of interest.

Copyright (c) 2016 Mende, Aylward, Eppley, Nielsen and DeLong. This is an openaccess article distributed under the terms of the Creative Commons Attribution License (CC BY). The use, distribution or reproduction in other forums is permitted, provided the original author(s) or licensor are credited and that the original publication in this journal is cited, in accordance with accepted academic practice. No use, distribution or reproduction is permitted which does not comply with these terms. 